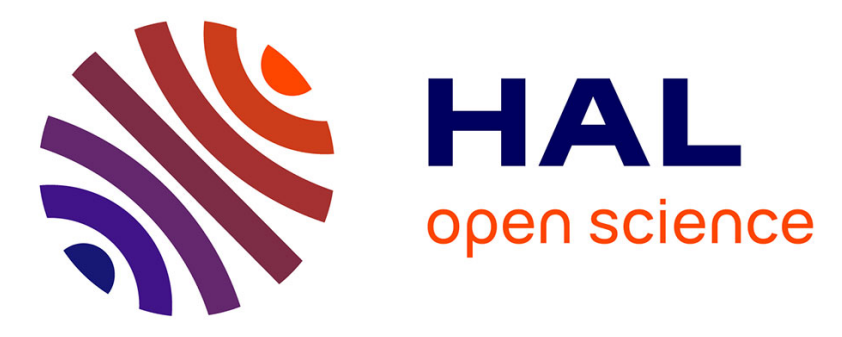

\title{
Sampled output observer design for a class of nonlinear systems
}

Mondher Farza, Ibtissem Bouraoui, Tomas Menard, Ridha Ben Abdennour, Mohammed M'Saad

\section{- To cite this version:}

Mondher Farza, Ibtissem Bouraoui, Tomas Menard, Ridha Ben Abdennour, Mohammed M'Saad. Sampled output observer design for a class of nonlinear systems. Control Conference (ECC), 2014 European, Jun 2014, strasbourg, France. pp.312 - 317, 10.1109/ECC.2014.6862309 . hal-01062548

\section{HAL Id: hal-01062548 \\ https://hal.science/hal-01062548}

Submitted on 31 Oct 2014

HAL is a multi-disciplinary open access archive for the deposit and dissemination of scientific research documents, whether they are published or not. The documents may come from teaching and research institutions in France or abroad, or from public or private research centers.
L'archive ouverte pluridisciplinaire HAL, est destinée au dépôt et à la diffusion de documents scientifiques de niveau recherche, publiés ou non, émanant des établissements d'enseignement et de recherche français ou étrangers, des laboratoires publics ou privés. 


\title{
Sampled output observer design for a class of nonlinear systems
}

\author{
M. Farza ${ }^{1}$, I. Bouraoui ${ }^{1,2}$, T. Ménard ${ }^{1}$, R. Ben Abdennour ${ }^{2}$, M. M'Saad $^{1}$
}

\begin{abstract}
This paper presents a continuous-discrete time observer for a class of uncertain nonlinear systems with non uniformly sampled measurements. Two features of the proposed observer are worth to be pointed out. The first one consists in the simplicity of its calibration, while the second one lies in its comprehensive convergence analysis. More specifically, it is shown that the observation error lies in a ball centered at the origin and whose radius is proportional to the bounds of the uncertainties and the sampling partition diameter. In the free uncertainties case, the exponential convergence to zero of the observation error is established under a well-defined condition on the maximum value of the sampling partition diameter. The effectiveness of the proposed observer is emphasized throughout simulation results involving the estimation of the reaction rate in a typical bioreactor.
\end{abstract}

Key words: Nonlinear systems, high gain observers, impulsive systems, continuous-discrete time observers, bioreactors, reaction rates estimation.

\section{INTRODUCTION}

Although a considerable research activity has been devoted to the observer design for nonlinear systems over the last decades, the available contributions deal mainly with continuous time measurements [6], [10], [5], [2], [14], [11]. The problem of sampled measurements has been particularly addressed by redesigning the available state observers that have been proposed for the continuous time measurements case [3], [8], [1], [12]. In all these contributions, a dynamical system, which is similar to the underlying system, is used to provide a state prediction over the sampling intervals. This state prediction is updated by the sampled measurements. An output predictor approach has been proposed in [9]. It consists in a continuous time observer incorporating a suitable predictor of the output over the sampling intervals. The output prediction is provided by the solution of an ordinary differential initialized with the sampled measurements. The underlying observer is a hybrid system which is able to recover the continuous time observer properties for relatively fast sampling. An impulsive continuous-discrete observer has been proposed in [13] using an appropriate LMI approach.

Research supported by the European Commission under the project HYCON2 Highly-complex and networked control systems. ${ }^{1}$ GREYC, UMR 6072 CNRS, Université de Caen, ENSICAEN 6 Bd Maréchal Juin, 14050 Caen Cedex, FRANCE. ${ }^{2}$ Unité de Recherche CONPRI, ENIG Gabès, Rue Omar Ibn El Khattab, 6029 Gabès, Tunisie
The contribution of this paper consists in designing a continuous-discrete time observer for a class of uncertain nonlinear systems with non uniformly sampled measurements from a redesigned version of the high gain observer proposed in [4] for continuous time measurements. Though, the proposed observer shares the impulsive nature of the observer given in [13] up to an adequate modification of the observer gain, it can be rewritten under the form of a hybrid system similar to the continuous-discrete time observer proposed in [9]. There are two main features of the proposed continuous-discrete time observer that are worth to be emphasized with respect to the available ones. The first one concerns the ease with which the observer gain is updated at sampling instants together with the simplicity of its implementation. The second feature is related to the convergence analysis simplicity and its ability to provide precise expressions of the upper bounds of the sampling partition diameter as well as the rate of the observation error convergence. More specifically, it is shown that the observation error lies in a ball centered at the origin with a radius proportional to the magnitude of the bounds of the uncertainties and the maximum sampling partition diameter. Moreover, it is shown that the ultimate bound of the observation error can be made arbitrarily small, as in the case where the output measurements are continuously available, when the maximum sampling partition diameter tends to zero. A particular emphasis is put on the fact that the observation error converges exponentially to the origin in the absence of uncertainties. Of particular interest, the expression of the underlying decay rate is given.

The paper is organized as follows. The observer design problem is stated in section 2 with a technical lemma that will be used to establish the main result of the paper. Section 3 is devoted to the main contribution of the paper. The fundamental result is first given with a comprehensive proof thanks to the technical lemma derived in section 2 . The effectiveness of the proposed observer is highlighted via simulation results in section 4. Some concluding remarks are given in section 5 .

Throughout the paper, $I_{p}$ and $0_{p}$ will denote the $\mathrm{p}$ dimensional identity and zero matrices respectively and $\|\cdot\|$ denotes the euclidian norm; $\lambda_{M}$ (resp. $\lambda_{m}$ ) is the maximum (resp. minimum) eigenvalue of a symmetric positive definite (SPD) matrix $P$ and $\sigma=\frac{\lambda_{M}}{\lambda_{m}}$ is the ratio of the maximum 
and minimum values of $P$.

\section{PROBLEM STATEMENT AND PRELIMINARIES}

\section{A. Presentation of the considered class of systems}

Consider the class of multivariable nonlinear systems that are diffeomorphic to the following block triangular form with specific uncertainties:

$$
\left\{\begin{aligned}
\dot{x}(t) & =A x(t)+\varphi(u(t), x(t))+B \varepsilon(t) \\
y\left(t_{k}\right) & =C x\left(t_{k}\right)=x^{1}\left(t_{k}\right)
\end{aligned}\right.
$$

with

$$
\begin{gathered}
x=\left(\begin{array}{c}
x^{1} \\
\vdots \\
x^{q-1} \\
x^{q}
\end{array}\right), \varphi(u, x)=\left(\begin{array}{c}
\varphi^{1}\left(u, x^{1}\right) \\
\varphi^{2}\left(u, x^{1}, x^{2}\right) \\
\vdots \\
\varphi^{q-1}\left(u, x^{1}, \ldots, x^{q-1}\right) \\
\varphi^{q}(u, x)
\end{array}\right) \\
A=\left(\begin{array}{cccc}
0_{p} & I_{p} & & 0_{p} \\
\vdots & \ddots & \ddots & \\
\vdots & & \ddots & I_{p} \\
0_{p} & 0_{p} & \ldots & 0_{p}
\end{array}\right), B=\left(\begin{array}{c}
0_{p} \\
\vdots \\
0_{p} \\
I_{p}
\end{array}\right) \\
C=\left(\begin{array}{llll}
I_{p} & \ldots & 0_{p} & 0_{p}
\end{array}\right)
\end{gathered}
$$

where $x \in \mathbb{R}^{n}$ is the state and $x^{i} \in \mathbb{R}^{p}$ for $i \in[1, q]$ are the state blocks, $u(t) \in U$ a compact subset of $\mathbb{R}^{m}$ denotes the system input and $y \in \mathbb{R}^{p}$ denotes the system output which is available only at the sampling instants satisfying $0 \leq t_{0}<\ldots<t_{k}<t_{k+1}<\ldots$ with time-varying sampling intervals $\tau_{k}=t_{k+1}-t_{k}$ and $\lim _{k \rightarrow \infty} t_{k}=+\infty$ and $\varepsilon: \mathbb{R}^{+} \mapsto \in \mathbb{R}^{p}$ is unknown function representing the system uncertainties and may depend on the state, the input and uncertain parameters.

As it is mentioned in the introduction, our main objective is to design a continuous-discrete time observer providing a continuous time estimation of the entire state of system (1) by using the output measurements that are available only at the sampling instants. The time intervals $\tau_{k}$ 's are naturally bounded away from zero by $\tau_{m}$ and are upperly bounded by the upper bound of the sampling partition diameter $\tau_{M}$, i.e.

$$
0<\tau_{m} \leq \tau_{k}=t_{k+1}-t_{k} \leq \tau_{M}, \quad \forall k \geq 0
$$

Such a design will be carried out under the following assumptions on the functions $\varphi^{i}$ and the unknown function $\varepsilon$.

A1. The functions $\varphi^{i}$ for $i \in[1, q]$ are globally Lipschitz with respect to $x$ uniformly in $u$, i.e. there exists $L>0$ such that for all $u \in U$ and for all $x, \bar{x} \in \mathbb{R}^{n}$, the following inequality holds for $i=1, \ldots, q$ :

$$
\left\|\varphi^{i}(u, x)-\varphi^{i}(u, \bar{x})\right\| \leq L\|x-\bar{x}\|
$$

A2. The unknown function $\varepsilon$ is bounded, i.e.

$$
\exists \delta>0 ; \forall t \geq 0:\|\varepsilon(t)\| \leq \delta
$$

There are two remarks that are worth to be pointed out on the considered class of systems. Firstly, it may seem very restrictive since it assumes a non prime dimension, i.e. $n=$ $p q$, and the state blocks $x^{k}$ have the same dimension $p$. This is by no means the case since it is shown in [7] that there exists a class of uniformly observable nonlinear systems that can be put under the form (1) via an injective map. Secondly, the Lipschitz assumption A1 which seems very restrictive can be relaxed in the case where the system state trajectory lies in a bounded set $\Omega$ by appropriately extending the nonlinearities $\varphi(u, x)$ as in [15], [2].

\section{B. A technical lemma}

In what follows, a technical lemma together with its proof is given. This lemma will be used to establish the main result of the paper in the next section.

Lemma 2.1: Consider a differentiable function $v: t \in$ $R^{+} \mapsto v(t) \in R^{+}$satisfying the following inequality

$$
\dot{v}(t) \leq-a v(t)+b \int_{t_{k}}^{t} v(s) d s+c \quad \forall t \in\left[t_{k}, t_{k+1}[\right.
$$

where $0<\tau_{m} \leq \tau_{k}=t_{k+1}-t_{k} \leq \tau_{M}<+\infty$ and $a, b$ and $c$ are positive reals satisfying

$$
\frac{b \tau_{M}}{a}<1
$$

Then, the function $v$ satisfies

with $\begin{gathered}v(t) \leq e^{-\eta\left(t-t_{0}\right)} v\left(t_{0}\right)+c \tau_{M}\left(1+\frac{1}{1-e^{-\eta \tau_{m}}}\right) \\ 0<\eta=\left(a-b \tau_{M}\right) e^{-a \tau_{M}}\end{gathered}$

Proof of lemma 2.1. We shall first prove that $v(t) \leq v\left(t_{k}\right)+$ $c\left(t-t_{k}\right) \quad \forall t \in\left[t_{k}, t_{k+1}\right.$ [ and use this property to establish the key inequality (8). By integrating (6) from $t_{k}$ to $t$, we obtain

$$
\begin{aligned}
v(t) & \leq v\left(t_{k}\right)-a \int_{t_{k}}^{t} v(s) d s+b \int_{t_{k}}^{t} \int_{t_{k}}^{s} v(\nu) d \nu d s+c\left(t-t_{k}\right) \\
& \leq v\left(t_{k}\right)+\left(b \tau_{M}-a\right) \int_{t_{k}}^{t} v(s) d s+c\left(t-t_{k}\right)
\end{aligned}
$$

And by using the condition (7), i.e. $b \tau_{M}-a<0$, the last inequality (10) becomes

$$
v(t) \leq v\left(t_{k}\right)+c\left(t-t_{k}\right)
$$

Now, we shall establish the inequality (8) using the property (11). Indeed, inequality (6) becomes

$$
\begin{aligned}
\dot{v}(t) & \leq-a v(t)+b \int_{t_{k}}^{t}\left(v\left(t_{k}\right)+c\left(s-t_{k}\right)\right) d s+c \\
& \leq-a v(t)+b\left(v\left(t_{k}\right)+c\left(t-t_{k}\right)\right) \int_{t_{k}}^{t} d s+c \\
& \leq-a v(t)+b \tau_{M}\left(v\left(t_{k}\right)+c\left(t-t_{k}\right)\right)+c
\end{aligned}
$$

Solving the above inequality from $t_{k}$ to $t$, we get

$$
\begin{aligned}
v(t) \leq & e^{-a\left(t-t_{k}\right)} v\left(t_{k}\right)+\left(b \tau_{M} v\left(t_{k}\right)+c\right) \int_{t_{k}}^{t} e^{-a(t-s)} d s \\
& +b \tau_{M} c \int_{t_{k}}^{t}\left(s-t_{k}\right) e^{-a(t-s)} d s \\
\leq & e^{-a\left(t-t_{k}\right)} v\left(t_{k}\right)+\frac{b \tau_{M} v\left(t_{k}\right)+c}{a}\left(1-e^{-a\left(t-t_{k}\right)}\right) \\
& +c a \int_{t_{k}}^{t}\left(s-t_{k}\right) e^{-a(t-s)} d s
\end{aligned}
$$


The last inequality results from condition (7), i.e. $b \tau_{M}<a$. Now, using an integration by part, one can show that

$$
a \int_{t_{k}}^{t}\left(s-t_{k}\right) e^{-a(t-s)} d s=\left(t-t_{k}\right)-\frac{1}{a}\left(1-e^{-a\left(t-t_{k}\right)}\right)
$$

And substituting (14) in (13), one gets

$$
\begin{aligned}
v(t) \leq & e^{-a\left(t-t_{k}\right)} v\left(t_{k}\right)+\frac{b \tau_{M} v\left(t_{k}\right)+c}{a}\left(1-e^{-a\left(t-t_{k}\right)}\right) \\
& +c\left(\left(t-t_{k}\right)-\frac{1}{a}\left(1-e^{-a\left(t-t_{k}\right)}\right)\right) \\
= & g(t) v\left(t_{k}\right)+c\left(t-t_{k}\right)
\end{aligned}
$$

where $g(t) \triangleq e^{-a\left(t-t_{k}\right)}\left(1-\frac{b \tau_{M}}{a}\right)+\frac{b \tau_{M}}{a}$. Let us show that $g(t) \leq e^{-\eta\left(t-t_{k}\right)}$ where $\eta$ is given as in (8). To this end, it suffices to show that $\delta(t)=\left(g(t)-e^{-\eta\left(t-t_{k}\right)}\right) \leq 0$ for all $t \geq t_{k}$. This can be done by simply showing that $\dot{\delta}(t) \leq 0$ since one can easily check that $\delta\left(t_{k}\right)=0$. This is actually the case since

$$
\begin{aligned}
\dot{\delta}(t) & =\dot{g}(t)+\eta e^{-\eta\left(t-t_{k}\right)} \\
& =-a e^{-a\left(t-t_{k}\right)}\left(1-\frac{b \tau_{M}}{a}\right)+\eta e^{-\eta\left(t-t_{k}\right)} \\
& \leq-e^{-a \tau_{M}}\left(a-b \tau_{M}\right)+\eta=0
\end{aligned}
$$

The last equality results from the expression of $\eta$ given by (8). Hence, inequality (16) becomes

$$
v(t) \leq e^{-\eta\left(t-t_{k}\right)} v\left(t_{k}\right)+c\left(t-t_{k}\right), \quad \forall t \in\left[t_{k}, t_{k+1}[, k \in \mathbb{N}\right.
$$

Iterating inequality (18), one can show that

$$
\begin{aligned}
v\left(t_{k}\right) \leq & e^{-\eta\left(\tau_{k-1}+\ldots+\tau_{0}\right)} v\left(t_{0}\right)+c\left(\tau_{k-1}+\tau_{k-2} e^{-\eta \tau_{k-1}}\right. \\
& \left.+\tau_{k-3} e^{-\eta\left(\tau_{k-1}+\tau_{k-2}\right)}+\ldots+\tau_{0} e^{-\eta\left(\tau_{k-1}+\ldots+\tau_{1}\right)}\right) \\
\leq & e^{-\eta\left(t_{k}-t_{0}\right)} v\left(t_{0}\right)+c\left(\tau_{k-1}+\tau_{k-2} e^{-\eta \tau_{k-1}}+\right. \\
& \left.\tau_{k-3} e^{-\eta\left(\tau_{k-1}+\tau_{k-2}\right)}+\ldots+\tau_{0} e^{-\eta\left(\tau_{k-1}+\ldots+\tau_{1}\right)}\right) \\
\leq & e^{-\eta\left(t_{k}-t_{0}\right)} v\left(t_{0}\right)+c \tau_{M}\left(\sum_{j=0}^{k-1} e^{-\eta j \tau_{m}}\right) \\
\leq & e^{-\eta\left(t_{k}-t_{0}\right)} v\left(t_{0}\right)+c \tau_{M}\left(\frac{1-e^{-\eta k \tau_{m}}}{1-e^{-\eta \tau_{m}}}\right) \\
\leq & e^{-\eta\left(t_{k}-t_{0}\right)} v\left(t_{0}\right)+c \tau_{M}\left(\frac{1}{1-e^{-\eta \tau_{m}}}\right)
\end{aligned}
$$

And using (19), inequality (18) becomes

$$
\begin{aligned}
v(t) & \leq e^{-\eta\left(t-t_{0}\right)} v\left(t_{0}\right)+c\left(t-t_{k}\right)+\frac{c \tau_{M}}{1-e^{-\eta \tau_{m}}} \\
& \leq e^{-\eta\left(t-t_{0}\right)} v\left(t_{0}\right)+c \tau_{M}\left(1+\frac{1}{1-e^{-\eta \tau_{m}}}\right)
\end{aligned}
$$

This ends the proof of the lemma.

\section{THE CONTINUOUS-DISCRETE TIME OBSERVER}

Let us consider the following impulsive system

$$
\begin{gathered}
\dot{\hat{x}}(t)=A \hat{x}(t)+\varphi(u(t), \hat{x}(t))-\theta \Delta_{\theta}^{-1} K e^{-\theta K^{1}\left(t-t_{k}\right)} \times \\
\left(C \hat{x}\left(t_{k}\right)-y\left(t_{k}\right)\right) \text { for } t \in\left[t_{k}, t_{k+1}[\right.
\end{gathered}
$$

where $\hat{x}=\left(\begin{array}{c}\hat{x}^{1} \\ \vdots \\ \hat{x}^{q}\end{array}\right)$ is the state estimate, $K=\left(\begin{array}{c}K^{1} \\ \vdots \\ K^{q}\end{array}\right)$ is a gain matrix with $K^{i}$ being $p \times p$ square matrices that are specified such that the matrix $\bar{A}=A-K C$ is Hurwitz and $\Delta_{\theta}$ is the block-diagonal matrix defined by:

$$
\Delta_{\theta}=\operatorname{diag}\left(I_{p}, \frac{1}{\theta} I_{p}, \ldots, \frac{1}{\theta^{q-1}} I_{p}\right)
$$

with $\theta \geq 1$. In the following, we shall show that this impulsive system is a continuous-discrete time observer for system (1) and exhibit its output prediction form.

\section{A. The convergence result}

The above impulsive system is actually a continuous-discrete time observer for system (1) as pointed out by the following result.

Theorem 3.1: Consider the system (1)-(2) subject to Assumption $\mathbf{A} 1$ and $\mathbf{A 2}$. Then, for every $u \in U$, for every $\theta>$ $\theta_{0}$, there exists $\chi_{\theta}>0, \eta_{\theta}\left(\tau_{M}\right)>0, N_{\theta}\left(\tau_{m}, \tau_{M}\right)>0$ such that if the upper bound of the sampling partition diameter $\tau_{M}$ is chosen such that $\tau_{M}<\chi_{\theta}$, then for every $\hat{x}(0) \in \mathbb{R}^{n}$, we have:

$\|\hat{x}(t)-x(t)\| \leq \lambda \theta^{q-1} e^{-\eta_{\theta}\left(\tau_{M}\right) t}\|\hat{x}(0)-x(0)\|+N_{\theta}\left(\tau_{m}, \tau_{M}\right) \delta$

where $x(t)$ is the unknown trajectory of (1) associated to the input $u, \hat{x}(t)$ is any trajectory of system (20) associated to $(u, y), \delta$ is the upper bound of $\|\varepsilon\|$ and the parameters $\lambda, \theta_{0}, \mu_{\theta}, M_{\theta}$ are given in the proof while $\tau_{m}$ and $\tau_{M}$ are defined in (3).

Proof of Theorem 3.1. Let $\tilde{x}(t)=\hat{x}(t)-x(t)$ be the observation error, it can easily be checked that

$\dot{\tilde{x}}(t)=A \tilde{x}+\Phi(u, \hat{x}, x)-\theta \Delta_{\theta}^{-1} K e^{-\theta K^{1}\left(t-t_{k}\right)} C \tilde{x}\left(t_{k}\right)-B \varepsilon(t)$

where $\Phi(u, \hat{x}, x)=\varphi(u, \hat{x})-\varphi(u, x)$. Setting $\bar{x}=\Delta_{\theta} \tilde{x}$ and using the following identities

$$
\Delta_{\theta} A \Delta_{\theta}^{-1}=\theta A \text { and } C \Delta_{\theta}^{-1}=C
$$

allow to get

$$
\begin{aligned}
\dot{\bar{x}}= & \Delta_{\theta} A \Delta_{\theta}^{-1} \bar{x}+\Delta_{\theta} \Phi(u, \hat{x}, x) \\
& -\theta K e^{-\theta K^{1}\left(t-t_{k}\right)} C \Delta_{\theta}^{-1} \bar{x}\left(t_{k}\right)-\Delta_{\theta} B \varepsilon(t) \\
=\theta A \bar{x}+\Delta_{\theta} \Phi(u, \hat{x}, x) & \\
\quad & \theta K e^{-\theta K^{1}\left(t-t_{k}\right)} C \bar{x}\left(t_{k}\right)-\frac{1}{\theta^{q-1}} B \varepsilon(t)
\end{aligned}
$$

Moreover, adding and subtracting the term $\theta K C \bar{x}$ in the last equation yields

$$
\begin{aligned}
\dot{\bar{x}}= & \theta(A-K C) \bar{x}+\Delta_{\theta} \Phi(u, \hat{x}, x) \\
& \quad+\theta K\left(C \bar{x}-e^{-\theta K^{1}\left(t-t_{k}\right)} C \bar{x}\left(t_{k}\right)\right)-\frac{1}{\theta^{q-1}} B \varepsilon(t) \\
=\theta \bar{A} \bar{x} & +\Delta_{\theta} \Phi(u, \hat{x}, x)+\theta K z-\frac{1}{\theta^{q-1}} B \varepsilon(t)
\end{aligned}
$$

where $z(t)=C \bar{x}(t)-e^{-\theta K^{1}\left(t-t_{k}\right)} C \bar{x}\left(t_{k}\right)=\bar{x}^{1}(t)-$ $e^{-\theta K^{1}\left(t-t_{k}\right)} \bar{x}^{1}\left(t_{k}\right)$. Notice that $z\left(t_{k}\right)=0$ and according to (23), the time derivative of $z$ can be written as follows

$$
\begin{aligned}
\dot{z}(t) & =\dot{\bar{x}}^{1}(t)+K^{1} \theta e^{-\theta K^{1}\left(t-t_{k}\right)} \bar{x}^{1}\left(t_{k}\right) \\
& =\theta \bar{x}^{2}+\Phi^{1}\left(u, \hat{x}^{1}, x^{1}\right)
\end{aligned}
$$


where $\Phi^{1}\left(u, \hat{x}^{1}, x^{1}\right)=\varphi^{1}\left(u, \hat{x}^{1}\right)-\varphi^{1}\left(u, x^{1}\right)$.

Now, let us consider the following candidate quadratic Lyapunov function: $V(\bar{x})=\bar{x}^{T} P \bar{x}$ where $P$ is defined by

$$
P \bar{A}+\bar{A}^{T} P \leq-2 \mu I_{n}
$$

Proceeding as in the continuous time case, one can show that

$$
\begin{gathered}
\dot{V}(\bar{x})=2 \bar{x}^{T}(t) P\left(\theta \bar{A} \bar{x}+\Delta_{\theta} \Phi(u, \hat{x}, x)+\theta K z\right) \\
-\frac{2}{\theta^{q-1}} \bar{x}^{T}(t) P B \varepsilon(t) \\
\leq-\left(2 \mu \theta-2 L \sqrt{n} \lambda_{M}\right)\|\bar{x}\|^{2}+2 \theta \bar{x}^{T} P K z \\
-\frac{2}{\theta^{q-1}} \bar{x}^{T}(t) P B \varepsilon(t)
\end{gathered}
$$

Choosing $\theta$ such that $2\left(\mu \theta-\sqrt{n} \lambda_{M} L\right)>\mu \theta$ i.e.

$$
\theta>\theta_{0}^{\prime} \triangleq \frac{2 L \sqrt{n} \lambda_{M}}{\mu}
$$

the last inequality (26) becomes

$$
\begin{gathered}
\dot{V}(\bar{x}) \leq-\frac{\mu \theta}{\lambda_{M}} V(\bar{x})+2 \theta\|P \bar{x}\|\|K\|\|z(t)\| \\
+\frac{2}{\theta^{q-1}}\|P \bar{x}\|\|\varepsilon(t)\| \\
\leq-\frac{\mu \theta}{\lambda_{M}} V(\bar{x})+2 \theta \sqrt{\lambda_{M}} \sqrt{V(\bar{x})}\|K\|\|z(t)\| \\
+\frac{2 \sqrt{\lambda_{M}}}{\theta^{q-1}} \delta \sqrt{V(\bar{x})}
\end{gathered}
$$

Furthermore, integrating equation (24) from $t_{k}$ to $t$ while using the fact that $z\left(t_{k}\right)=0$ yields

$$
z(t)=\int_{t_{k}}^{t}\left(\theta \bar{x}^{2}(s)+\Phi^{1}\left(u(s), \hat{x}^{1}(s), x^{1}(s)\right)\right) d s
$$

Bearing in mind that $\Phi^{1}\left(u, \hat{x}^{1}, x^{1}\right)=\varphi^{1}\left(u, \hat{x}^{1}\right)-\varphi^{1}\left(u, x^{1}\right)$, one gets

$$
\begin{aligned}
\|z(t)\| & \leq \int_{t_{k}}^{t}\left(\theta\left\|\bar{x}^{2}(s)\right\|+L\left\|\bar{x}^{1}(s)\right\|\right) d s \\
& \leq(\theta+L) \int_{t_{k}}^{t}\|\bar{x}(s)\| d s \leq \frac{\theta+L}{\sqrt{\lambda_{m}}} \int_{t_{k}}^{t} \sqrt{V(\bar{x}(s))} d s
\end{aligned}
$$

Combining the above inequalities, one obtains

$$
\begin{gathered}
\dot{V}(\bar{x}(t)) \leq-\frac{\mu \theta}{\lambda_{M}} V(\bar{x}(t))+ \\
2 \theta \sqrt{\frac{\lambda_{M}}{\lambda_{m}}}\|K\|(\theta+L) \sqrt{V(\bar{x}(t))} \int_{t_{k}}^{t} \sqrt{V(\bar{x}(s))} d s \\
+\frac{2 \sqrt{\lambda_{M}}}{\theta^{q-1}} \delta \sqrt{V(\bar{x})}
\end{gathered}
$$

or equivalently

$$
\begin{aligned}
& \frac{d}{d t} \sqrt{V(\bar{x}(t))} \leq-\frac{\mu \theta}{2 \lambda_{M}} \sqrt{V(\bar{x}(t))}+ \\
&+\theta \sqrt{\sigma}\|K\|(L+\theta) \int_{t_{k}}^{t} \sqrt{V(\bar{x}(s))} d s \\
&+\frac{\sqrt{\lambda_{M}}}{\theta^{q-1}} \delta
\end{aligned}
$$

where $\sigma=\frac{\lambda_{M}}{\lambda_{m}}$. Finally, let

$$
a_{\theta}=\frac{\mu \theta}{2 \lambda_{M}}, b_{\theta}=\theta \sqrt{\sigma}\|K\|(L+\theta) \text { and } c_{\theta}=\frac{\sqrt{\lambda_{M}}}{\theta^{q-1}} \delta
$$

and assume that the upper diameter of the sampling partition $\tau_{M}$ satisfies the following condition $\tau_{M}<\frac{a_{\theta}}{b_{\theta}}$, i.e.

$\frac{b_{\theta} \tau_{M}}{a_{\theta}}<1$ or more explicitly $2 \tau_{M}(L+\theta) \frac{\|K\| \lambda_{M} \sqrt{\sigma}}{\mu}<1$

Then, according to Lemma 1, one has

$\sqrt{V(\bar{x}(t))} \leq e^{-\eta_{\theta} t} \sqrt{V(\bar{x}(0))}+c_{\theta} \tau_{M}\left(1+\frac{1}{1-e^{-\eta_{\theta} \tau_{m}}}\right)$

where

$$
0<\eta_{\theta}\left(\tau_{M}\right)=\left(a_{\theta}-b_{\theta} \tau_{M}\right) e^{-a_{\theta} \tau_{M}}
$$

Note that the constant $\chi_{\theta}$ involved in the theorem is $\chi_{\theta}=\frac{a_{\theta}}{b_{\theta}}=\frac{\mu}{2(L+\theta)\|K\| \lambda_{M} \sqrt{\sigma}}$. Now, coming back to the original coordinates of the observation error $\tilde{x}$ and proceeding as in the continuous time measurements case, we get

$$
\begin{aligned}
\|\tilde{x}(t)\| \leq & \sqrt{\sigma} \theta^{q-1} e^{-\eta_{\theta} t}\|\bar{x}(0)\| \\
& +\frac{\theta^{q-1}}{\sqrt{\lambda_{m}}} c_{\theta} \tau_{M}\left(1+\frac{1}{1-e^{-\eta_{\theta}\left(\tau_{M}\right) \tau_{m}}}\right)
\end{aligned}
$$

Substituting in the above inequality $c_{\theta}$ by its expression (29) leads to

$$
\begin{aligned}
\|\tilde{x}(t)\| \leq & \sqrt{\sigma} \theta^{q-1} e^{-\eta_{\theta}\left(\tau_{M}\right) t}\|\bar{x}(0)\| \\
& +\sqrt{\sigma} \tau_{M}\left(1+\frac{1}{1-e^{-\eta_{\theta}\left(\tau_{M}\right) \tau_{m}}}\right) \delta
\end{aligned}
$$

The functions $\eta_{\theta}\left(\tau_{M}\right)$ and $N_{\theta}\left(\tau_{m}, \tau_{M}\right)$ involved in theorem 3.1 are hence given by

$$
\begin{aligned}
& \quad \eta_{\theta}\left(\tau_{M}\right)=\left(a_{\theta}-b_{\theta} \tau_{M}\right) e^{-a_{\theta} \tau_{M}} \\
& \text { and } N_{\theta}\left(\tau_{m}, \tau_{M}\right)=\sqrt{\sigma} \tau_{M}\left(1+\frac{1}{1-e^{-\eta_{\theta}\left(\tau_{M}\right) \tau_{m}}}\right)
\end{aligned}
$$

where $a_{\theta}$ and $b_{\theta}$ are given by (29). This completes the proof of theorem 3.1.

Remark 3.1: In order to emphasize the relationship between the rate of the exponential decreasing to zero and the ultimate bound of the observation error given by the continuous case (see [4]) and the theorem 3.1 for the continuous-discrete time case, we shall consider the case where the sampling period is constant and investigate the behaviour of the exponential decay rate and the ultimate bound. Indeed, one has

$$
\begin{aligned}
\eta_{\theta}\left(T_{s}\right) & \triangleq\left(a_{\theta}-b_{\theta} T_{s}\right) e^{-a_{\theta} T_{s}} \\
N_{\theta}\left(T_{s}\right) & \triangleq T_{s} \sqrt{\sigma}\left(1+\frac{1}{1-e^{-\eta_{\theta}\left(T_{s}\right) T_{s}}}\right)
\end{aligned}
$$

It is easy to check that for all $T_{s} \geq 0$ satisfying (30), the function $\eta_{\theta}\left(T_{s}\right)$ is a decreasing function of $T_{s}$ and $N_{\theta}\left(T_{s}\right)$ is an increasing function of $T_{s}$. Now, let us take the limits of these functions when $T_{s}$ tends to zero. Indeed, one has:

$$
\begin{aligned}
\lim _{T_{s} \rightarrow 0} \eta_{\theta}\left(T_{s}\right) & =a_{\theta}=\frac{\mu \theta}{2 \lambda_{M}}=\mu_{\theta} \\
\lim _{T_{s} \rightarrow 0} N_{\theta}\left(T_{s}\right) & =\lim _{T_{s} \rightarrow 0} T_{s} \sqrt{\sigma}\left(1+\frac{1}{1-e^{-\eta_{\theta}\left(T_{s}\right) T_{s}}}\right) \\
& =\lim _{T_{s} \rightarrow 0} T_{s} \sqrt{\sigma}\left(1+\frac{1}{T_{s} \eta_{\theta}\left(T_{s}\right)}\right)=\frac{\sqrt{\sigma}}{\mu_{\theta}}=M_{\theta}
\end{aligned}
$$


where $\mu_{\theta}$ and $M_{\theta}$ are respectively the exponential decay rate and the function intervening in the ultimate bound expression obtained in the continuous output case. So, the results obtained in the sampled output case are quite coherent with those derived in the continuous output case: the decreasing to zero of the observation error is inversely proportional to the magnitude of the sampling period while the value of the ultimate bound is proportional to this magnitude. Moreover, when the sampling period tends to zero, we meet the same expressions for the decay rate and ultimate bound as those derived in the continuous output case.

\section{EXAMPLE}

We consider a simple microbial culture which involves a single biomass $X$ growing on a single substrate $S$. The bioprocess is operating in a continuous mode with a timevarying dilution rate $D(t)$ and a constant input substrate concentration $S_{i n}$. The underlying mathematical dynamical model of the process is

$$
\left\{\begin{aligned}
\dot{X} & =r-D X \\
\dot{S} & =-k r+D\left(S_{i n}-S\right)
\end{aligned}\right.
$$

where $X$ and $S$ are the respective concentrations of the considered species, $r$ is the reaction rate and $k$ is a yield coefficient. The reaction rate $r$ is generally a very complex function of the operating conditions and the state of the process. The analytical modelling of this function is often cumbersome and still constitutes a challenging problem. To tackle this problem, this functions is treated as a time-varying parameter that needs to be estimated from the biomass concentration. Such an estimation shall be provided by an impulsive continuous-discrete time observer of the form (20) designed by considering the following rate estimation model

$$
\left\{\begin{array}{cl}
\dot{X}(t) & =r(t)-D X \\
\dot{r}(t) & =\varepsilon(t) \\
y\left(k T_{s}\right) & =X\left(k T_{s}\right) k \in \mathbb{N}
\end{array}\right.
$$

The equations of the impulsive continuous-discrete time observer specialize as follows:

$$
\left\{\begin{aligned}
\dot{\hat{X}}(t)= & \hat{r}(t)-D(t) \hat{X}(t) \\
& -2 \theta e^{-2\left(t-k T_{s}\right)}\left(\hat{X}\left(k T_{s}\right)-X\left(k T_{s}\right)\right) \\
\dot{\hat{r}}(t)= & -\theta^{2} e^{-2\left(t-k T_{s}\right)}\left(\hat{X}\left(k T_{s}\right)-X\left(k T_{s}\right)\right)
\end{aligned}\right.
$$

The gain $K$ of the observer (20)is chosen as $K=\left[\begin{array}{ll}2 & 1\end{array}\right]^{T}$ in order to assign all the modes of the matrix $A-K C$ at $(-1)$.

The simulation experiments have been performed using the following model for the reaction rate

$$
r=\frac{\mu^{\star} S X}{k_{C} X+S}
$$

where $\mu^{\star}$ and $K_{C}$ are constant kinetic parameters. The values of the kinetic parameters and the initial conditions have been respectively given by

$\mu^{\star}=1 / 6 h^{-1}, k=1 g \cdot g^{-1}, K_{C}=1 g \cdot g^{-1}, S_{i n}=1.3158 g \cdot l^{-1}$

and

$X(0)=\hat{X}(0)=1 g \cdot l^{-1}, S(0)=0.3158 g \cdot l^{-1}, \hat{r}(0)=0 g \cdot(l . h)^{-1}$
The dilution rate that has been considered is shown in figure 1a, i.e. a trapezoidal signal varying from 0.0 .4 to $0.12 h^{-1}$. In order to simulate practical situations, all the simulation experiments have been carried out with noisy data measurements: the measurements of $X$ issued from the simulation of system (37)-(39) are corrupted by an additive gaussian noise with zero mean value and a standard deviation equal to 0.0316 .

The simulation study has been performed as follows. First, several simulation experiments have been carried out assuming continuous time output measurements. This allows to obtain a lower bound on the design parameter $\theta$ for which the underlying continuous time observer performs well i.e. a good compromise between an admissible tracking of the kinetic parameter variation and a good behavior of the observer with respect to noise measurements. The value of $\theta$ ensuring such a compromise was about 1.5. Then, an other set of simulation experiments has been performed with this fixed design parameter value $(\theta=1.5)$ and different values for the sampling period. From an experiment to the other, the value of the sampling period was constant, $\tau_{M}=T_{s}$, and has been increased until reaching the maximum allowable value $\tau_{M}$ for which the performance of the continuous-discrete time observer still be quite satisfactory. The obtained value was about $\tau_{M}=1.1 \mathrm{~h}$. We have reproduced in figure 1e three sets of results corresponding to the estimation of the reaction rate $r$ obtained with the same value of the design parameter $\theta=1.5$. The first set provides the estimate of $r$ in the case of continuous time measurements and it is obtained by simulating an observer given in [4]. This estimate can serve as a reference (the ideal case) for the estimates obtained with the continuous-discrete time observer (39). The second and third sets are obtained with $T_{s}=0.05 h$ and $T_{s}=1 h$, respectively. As expected from the theoretical analysis, the best estimates are obtained with smaller values of the sampling period. Moreover, for relatively low values of $T_{s}$ (e.g. $T_{s}=0.05 h$ ), the obtained estimates are quite similar to those obtained with continuous measurements.

It is worth noticing that the expressions of the reactions rates, introduced for simulation purposes and in order to obtain the pseudo-measurements of $X$ and $P$, are not known by observer (39).

\section{CONCLUSion}

A high gain observer has been derived for a class of nonlinear systems with sampled output measurements involving specific uncertainties. This observer provides continuous time estimates of the whole system state from the available output samples. The underlying observation error convergence has been established under a well defined condition on the observer, the system parameter and the maximum allowable value of the sampling partition diameter. More specifically, it 


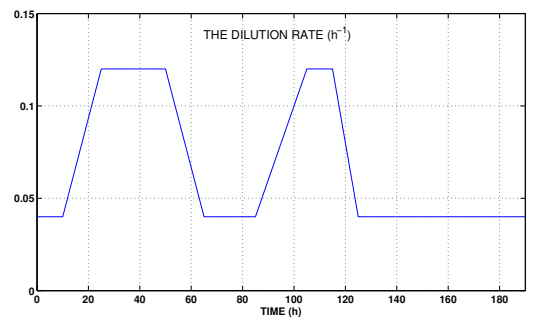

(a) Time evolution of the dilution rate

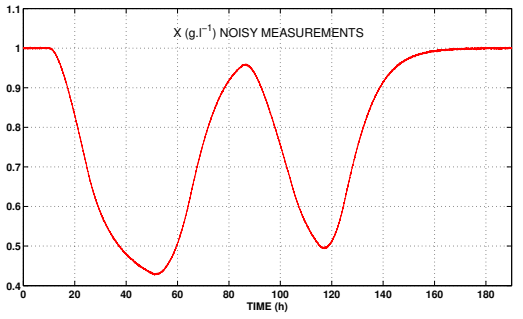

(b) Continuous noisy measurements of $X$

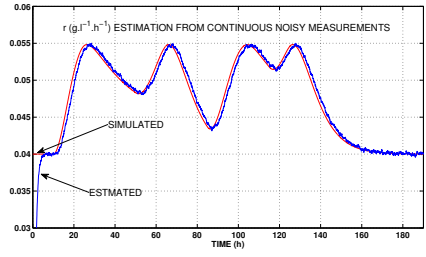

(c) Estimation of $r$ (i)

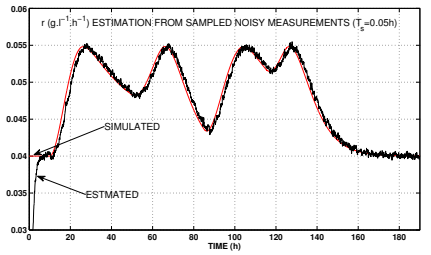

(d) Estimation of $r$ (ii)

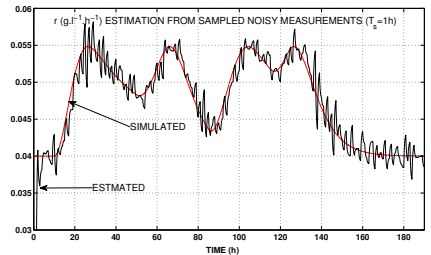

(e) Estimation of $r$ (iii) has been shown that the observation error remains confined in a ball centered at the origin with a radius proportional to the magnitudes of the uncertainties and the maximum of the sampling partition diameter. A particular emphasis has been put on the observation error exponential convergence to zero in the uncertainties free case. Of practical interest, it has been shown that the proposed observer can be used to perform an on-line relatively accurate estimation of the reaction rate in biochemical reactors.

\section{REFERENCES}

[1] V. Andrieu and M. Nadri. Observer design for lipschitz systems with discrete-time measurements. In In Proc. 49th IEEE Conference on Decision and Control, Atlanta, Georgia USA, 2010.

[2] V. Andrieu and L. Praly. On the existence of kazantzis-kravaris / Luenberger observers. SIAM J. Control and Optimization, 45:432456, 2006.

[3] F. Deza, E. Busvelle, J.P. Gauthier, and D. Rakotopara. High gain estimation for nonlinear systems. Systems \& Control Letters, 18:295299, 1992.

[4] M. Farza, M. M'Saad, and L. Rossignol. Observer design for a class of MIMO nonlinear systems. Automatica, 40(1):135-143, 2004.

[5] M. Farza, M. M'Saad, M. Triki, and T. Maatoug. High gain observer for a class of non-triangular systems. Systems \& Control Letters, 60(1):27-35, 2011.

[6] J.P. Gauthier and I. Kupka. Deterministic Observation Theory and Applications. Cambridge University Press, 2001.

[7] H. Hammouri and M. Farza. Nonlinear observers for locally uniformly observable systems. ESAIM J. on Control, Optimisation and Calculus of Variations, 9:353-370, 2003.

[8] H. Hammouri, M. Nadri, and R. Mota. Constant gain observer for continuous-discrete time uniformly observable systems. In Proc. of the the 45th IEEE Conference on Decision and Control, San Diego, CA, USA, 2006.

[9] I. Karafyllis and C. Kravaris. From continuous-time design to sampleddata design of observers. IEEE Transactions on Automatic Control, 54(9):2169-2174, 2009.

[10] N. Kazantzis and C. Kravaris. Nonlinear observer design using Lyapunov's auxiliary theorem. Systems \& Control Letters, 34:241247, 1998
[11] A. J. Krener and A. Isidori. Linearization by output injection and nonlinear observers. Syst. Contr. Lett., 3:47-52, 1983.

[12] M. Nadri, H. Hammouri, and R.M. Grajales. Observer design for uniformly observable systems with sampled measurements. IEEE Transactions on Automatic Control, 58:757-762, 2013.

[13] T. Raff, M. Kögel, and F. Allgöwer. Observer with sample-andhold updating for Lipschitz nonlinear systems with nonuniformly sampled measurements. In Proc. of the American Control Conference, Washington,USA, 2008.

[14] R. Rajamani. Observers for Lipschitz Nonlinear Systems. IEEE Transactions on Automatic Control, 43(3):397-401, 1998.

[15] H. Shim, Y. I. Son, and J. H. Seo. Semi-global observer for multioutput nonlinear systems. Systems and Control Letters, 42:233-244, 2001. 